\title{
SÍNDROME METABÓLICO: INTERVENCIONES EN EL ESTILO DE VIDA
}

\author{
METABOLIC SYNDROME: LIFESTYLE INTERVENTION
}

Las complicaciones vasculares propias de la diabetes pueden aparecer aún en etapas tempranas como la intolerancia a la glucosa en ayunas o postprandial. Por lo tanto el screening regular y la detección temprana de estas complicaciones tienen un alto significado clínico.

La obesidad central o visceral comúnmente se asocia a un conjunto de factores de riesgo (hipertensión, disglucemia, dislipemia) que aumenta el riesgo de padecer diabetes y enfermedad cardiovascular; precisamente el término síndrome metabólico (SM) se usa para describir esta asociación de factores ${ }^{2}$.

La neuropatía autonómica cardiovascular (NAC) es la forma clínica más estudiada de las neuropatías autonómicas y diversos trabajos han demostrado su relación con la isquemia miocárdica silente e incluso un aumento de cuatro veces en el riesgo de mortalidad cardiovascular. A su vez la obesidad visceral ha sido mencionada en distintos trabajos con una marcada correlación con alteraciones propias de la disautonomía cardiovascular ${ }^{3}$.

La obesidad se ha asociado en modelos animales a NAC y en humanos con aumento de la actividad simpática y con efecto inverso sobre la actividad parasimpática ${ }^{3}$.

Algunos autores especulan que el nexo de unión entre la obesidad y la NAC es la insulinorresistencia a través de la sobre estimula- ción del sistema simpático y la disminución del parasimpático 4 .

La obesidad se ha vinculado a la alteración en la variabilidad de la frecuencia cardíaca, sin embargo hay pocos estudios que evalúen el efecto de la pérdida de peso en NAC.

Precisamente el estudio publicado en este número por la Dra. Graciela Fuente y col. es un trabajo de intervención nutricional de un año de duración con el objetivo de reducción del $10 \%$ del peso corporal en pacientes con síndrome metabólico y evaluando los test de Ewing a través de un programa informático.

En los pacientes que llegan a cumplir el objetivo, se demostró una reducción significativa en todos los componentes del SM, inclusive el $93,7 \%$ de los intolerantes a la glucosa llegó a la euglucemia. La NAC mejoró en forma significativa en el $62 \%$ de los pacientes.

Este elegante trabajo -y muy prolijamente diseñado por los autores- demuestra la necesidad de poner énfasis en la intervención en el estilo de vida, en este caso el descenso de peso, para mejorar todos los parámetros del SM y lo que es tan importante la neuropatía autonómica cardiovascular que tantas implicancias puede tener en complicaciones cardiovasculares y cuyas opciones terapéuticas son muy escasas. 


\section{BIBLIOGRAFÍA}

1. Ko SH, et al. Cardiovascular autonomic dysfunction predicts acute ischemic stroke in patients with type 2 diabetes mellitus: a 7-year follow-up study. Diabetic Medicine 2008; 25:1171-1177.

2. Fitcett $D$, et al. The metabolic syndrome is an important concept in therapeutic decisicion-making. Canadian Journal of Cardiology 2015; 31:596-600.
3. Jang EH, et al. Influence of visceral adipocity on cardiovascular autonomic neuropathy in patients with type 2 diabetes mellitus. Diabetes Metab J 2012; 36:285-292.

4. Grewal S, et al. Effect of obesity on autonomic nervous system. Int J Bio Med Sci 2011; 1 (2):15-18.

5. Damodaran A, et al. Autonomic dysfunction in central obesity. World Journal of Medical Sciencies 2013; 8(2): 118-122. 